\title{
The Algebra of Weyl Symmetrised Polynomials and Its Quantum Extension
}

\author{
I. M. Gelfand ${ }^{\star}$ and D. B. Fairlie ${ }^{\star \star, \star \star \star \star}$ \\ Harvard University, Cambridge, MA 02138, USA \\ Received May 29, 1990; in revised form July 25, 1990
}

\begin{abstract}
The Algebra of Weyl symmetrised polynomials in powers of Hamiltonian operators $P$ and $Q$ which satisfy canonical commutation relations is constructed. This algebra is shown to encompass all recent infinite dimensional algebras acting on two-dimensional phase space. In particular the Moyal bracket algebra and the Poisson bracket algebra, of which the Moyal is the unique one parameter deformation are shown to be different aspects of this infinite algebra. We propose the introduction of a second deformation, by the replacement of the Heisenberg algebra for $P, Q$ with a $q$-deformed commutator, and construct algebras of $q$-symmetrised Polynomials.
\end{abstract}

\section{Introduction}

In the first section of this article we present an account of some ideas, dating back to the pioneering work of Hermann Weyl which generalise the algebra of functions on a classical phase space to a space co-ordinatised by canonically quantised operators. These ideas have surfaced from time to time in the literature $[1,2,3]$ and are now again very much alive in considerations of area preserving diffeomorphisms of two-dimensional manifolds, geometric quantisation and large $N$ limits of $S U(N)[4,5,6]$. The fundamental algebraic structure which we study is the algebra of symmetrised, averaged polynomials in $P$ and $Q$, where $P, Q$ satisfy the canonical commutation relations of the Heisenberg algebra;

$$
P Q-Q P=i \lambda .
$$

Here we take $\lambda$ as a numerical constant, but bear in mind that for possible novel

\footnotetext{
* On leave from Moscow State University

$\star \star$ On Research Leave from the University of Durham, U.K. (Present Address)

$\star \star \star$ Research supported in part by the Department of Energy under Grant DE/FG02/88/ ER25065, and by a grant from the Alfred P. Sloan Foundation and the Fulbright Commission
} 
physical applications of the results of the later sections that $\lambda$ should be accorded the dimensions of Planck's constant $h$, i.e. the dimensions of action. We restrict our considerations to a two-dimensional phase space; the generalization is straightforward.

In the second section we connect this algebra with the Moyal deformation of the Poisson bracket, which is related to an associative, non-abelian product on function space on a symplectic manifold. This construction is realised through a non-canonical transformation of $P, Q$ in terms of a two-dimensional manifold and its tangent space. Two special limits are considered; one where $\lambda=\frac{\pi}{M}, M$ integral, and $\lambda \rightarrow 0$. In the former case the algebra is shown to reduce to a group bundle with fiber $U(M)$ over the torus; in the latter with the Poisson algebra of area preserving transformations of two-dimensional manifolds. In sections three, four and five these constructions are subjected to a further deformation; the Heisenberg algebra (1) is replaced by the $q$-deformation

$$
P Q-q Q P=i \lambda
$$

and the analysis of sections one and two is repeated, this time including the $q$ deformation parameter. Representations of the $q$-Heisenberg algebra are analysed.

\section{The Algebra of Weyl-Ordered Operators}

Define $T_{j, m}$ as the fully symmetrised, averaged, sum of monomials of degree $j$ in $P$, and $m$ in $Q$, i.e.

$$
\begin{aligned}
T_{1,1} & =\frac{P Q+Q P}{2}, \\
T_{3,1} & =\frac{P^{3} Q+P^{2} Q P+P Q P^{2}+Q P^{3}}{4} \text { etc. }
\end{aligned}
$$

Note that $T_{j, m}$ depends implicitly upon $\lambda$, and may be derived from the generating function:

$$
(a P+b Q)^{s}=\sum_{j=0}^{s}\left(\begin{array}{l}
s \\
j
\end{array}\right) a^{j} b^{s-j} T_{j, s-j}
$$

Theorem. The commutator $\left[T_{j, m}, T_{k, n}\right]$ for $j, m \geqq 1$ is given by the following expression:

$$
\begin{aligned}
{\left[T_{j, m}, T_{k, n}\right]=} & \sum_{r=0}^{\min \{[(j+k-1) / 2],[(m+n-1) / 2]\}} \frac{(2 i \lambda)^{2 r+1}}{(2 r+1) !} \\
& \cdot \sum_{s=0}^{2 r+1}(-1)^{s}\left(\begin{array}{c}
2 r+1 \\
s
\end{array}\right) \frac{j ! k ! m ! n !}{(j+s-2 r-1) !(k-s) !(m-s) !(n+s-2 r-1) !} \\
& \cdot T_{j+k-2 r-1, m+n-2 r-1} .
\end{aligned}
$$


Proof. Consider instead the commutator of the operators

$$
\begin{gathered}
E_{a, b}=\frac{1}{2 i \lambda} \exp \sqrt{2 i}(a P+b Q), \\
{\left[E_{a, b}, E_{c, d}\right]=-\frac{1}{\lambda} \sin \lambda(a d-b c) E_{a+c, b+d} .}
\end{gathered}
$$

This follows from the Baker-Campbell-Hausdorff formula:

$$
\exp (A+B)=\exp B \exp \frac{[A, B]}{2} \exp A
$$

for operators $A, B$ such that $[A,[A, B]]=[B,[A, B]]=0$, by choice of $A=\sqrt{2 i}(a P+b Q), B=\sqrt{2 i}(c P+d Q)$. Now observe that the coefficient of $a^{j} b^{m}$ in $\exp \sqrt{2}(a P+b Q)$ is simply $\frac{\left(2^{(j+m) / 2}\right)}{j ! m !} T_{j, m}$. Then by comparison of coefficients of $a^{j} b^{m} c^{k} d^{n}$ on both sides of Eq. (7) the result follows. (The factorials in the numerator of (5) arise from the normalisations of the left-hand side; those in the denominator come from a combination of normalisation factors and binomial coefficients in selecting the coefficient of $a^{j-2 r-1+s} b^{m-s} c^{k-s} d^{k-2 r-1+s}$ from the expansion of $\exp \sqrt{2}(a P+b Q)$.)

This is the algebra quoted by C. M. Bender and G. Dunne [7] in the particular case where $j, k, m, n \geqq 0$. Equation (7) may be interpreted as an algebra of vertex operators, and if $a, b \in \mathbf{Z}$ the two limits $\lambda=\frac{\pi}{M}, M$ integral and $\lambda \rightarrow 0$ are particularly interesting [4]. Consider the algebra (7) modulo the equivalence relation

$$
E_{a+M s_{1}, b+M s_{2}}-\exp \left(\mu_{1} s_{1}+\mu_{2} s_{2}\right) E_{a, b}=0, \quad 0 \leqq \mu_{1}, \mu_{2} \leqq 2 \pi
$$

Theorem. For every point $\left(\mu_{1}, \mu_{2}\right)$ of the torus there is a factor algebra which is isomorphic to $U(M)$.

Proof. The algebra $U(M)$ is generated by the operators $J_{a, b}=\omega^{a b / 2} g^{a} h^{b}$, where $g, h$ satisfy the relations;

$$
\begin{aligned}
h g=\omega g h, \quad g^{M}=h^{M} & =1, \\
\text { where } \quad \omega^{M} & =1,
\end{aligned}
$$

since a faithful representation of (8) is realised by the following matrices and their products, which span the space of $U(M)$ :

$$
g=\left(\begin{array}{ccccc}
1 & \cdot & \cdot & \cdots & \cdot \\
\cdot & \omega & \cdot & \cdots & \cdot \\
\cdot & \cdot & \omega^{2} & \cdots & \cdot \\
\vdots & \vdots & \vdots & \ddots & \vdots \\
\cdot & \cdot & \cdot & \cdots & \omega^{N-1}
\end{array}\right) ; h=\left(\begin{array}{ccccc}
\cdot & 1 & \cdot & \cdots & \cdot \\
\cdot & \cdot & 1 & \cdots & \cdot \\
\vdots & \vdots & \vdots & \cdot & \vdots \\
\cdot & \cdot & \cdot & \cdots & 1 \\
\pm 1 & \cdot & \cdot & \cdots & \cdot
\end{array}\right)
$$

(The sign in the lower left element of $h$ is taken as positive or negative, according as $M$ is odd or even to give a positive determinant.) The Lie algebra of the $J_{a, b}$ is 
found to be

$$
\left[J_{a, b}, J_{c, d}\right]=\frac{i M}{\pi} \sin \frac{\pi}{M}(a d-b c) J_{a+c, b+d} .
$$

This is the factor algebra (9).

\section{Moyal Bracket}

There is another formalism which reproduces the same results: There is a deformation of the Poisson bracket algebra which involves the Moyal, or since bracket; $[1,2]$, defined for two $C^{\infty}$ functions $f(x, y), g(x, y)$ by

$$
\sin \lambda\{f, g\}=\sum_{p=0}^{\infty}(-1)^{p} \frac{\lambda^{2 p+1}}{(2 p+1) !} \sum_{k=0}^{2 p+1}(-1)^{k}\left(\begin{array}{c}
2 p+1 \\
k
\end{array}\right)\left(\partial_{x}^{k} \partial_{y}^{2 p+1-k} f\right)\left(\partial_{x}^{2 p+1-k} \partial_{y}^{k} g\right) .
$$

In fact the exponential bracket of Baker [1] of which the Moyal bracket is the imaginary part is a more fundamental concept. It is similarly defined by

$$
\exp i \lambda\{f, g\}=\sum_{p=0}^{\infty} \frac{(i \lambda)^{p}}{p !} \sum_{k=0}^{p-1}(-1)^{k}\left(\begin{array}{c}
p-1 \\
k
\end{array}\right)\left(\partial_{x}^{k} \partial_{y}^{p-k-1} f\right)\left(\partial_{x}^{p-k-1} \partial_{y}^{k} g\right) \text {. }
$$

It is the unique non-associative product on the space of $C^{\infty}$ functions of two variables such that the infinitesimal deformation is Poisson [8]. This concept are readily extended to a base space which is a $2 N$ dimensional symplectic manifold, as indeed is done in Baker's original paper [1]. The Moyal bracket algebra is given by

where

$$
\left[K_{f}, K_{g}\right]=i K_{\sin \lambda\{f, g\}}
$$

$$
K_{f}=\frac{1}{2} f\left(x-i \lambda \partial_{y}, y+i \lambda \partial_{x}\right)
$$

The above construction of $K_{f}$ is to be interpreted as the formal Taylor expansion of $\frac{1}{2} f\left(x-i \lambda \partial_{y}, y+i \lambda \partial_{x}\right)$ in powers of the partial derivatives, which are assumed to act on functions on the right; i.e.

$$
K_{f}=\frac{1}{2} \sum_{j=0} \sum_{k=0} \lambda^{j+k} \frac{(-i)^{j} i^{k}}{j ! k !}\left(\partial_{x}^{j} \partial_{y}^{k} f\right) \partial_{x}^{k} \partial_{y}^{j}
$$

Theorem. The algebras (7) and (15) are isomorphic.

Proof. Consider the following representation;

$$
P=\frac{i}{\sqrt{2}}\left(x-i \lambda \frac{\partial}{\partial y}\right), \quad Q=\frac{i}{\sqrt{2}}\left(y-i \lambda \frac{\partial}{\partial x}\right)
$$

which preserves the commutation relations (1). Since the generating function may be re-expressed in terms of the variables $x, y, \frac{\partial}{\partial x}, \frac{\partial}{\partial y}$ using the normal ordering rule as 


$$
(a P+b Q)^{s}=\left(\frac{i}{\sqrt{2}}\right)^{s} \sum_{j=0}^{s}\left(\begin{array}{l}
s \\
j
\end{array}\right)(a x+b y)^{j}(i \lambda)^{s-j}\left(-a \frac{\partial}{\partial y}+b \frac{\partial}{\partial x}\right)^{s-j},
$$

by comparison of coefficients of $a^{j} b^{s-j}$ on the right-hand side of (4) and (18) a differential operator realisation of $T_{j, m}$ may be found. This is given by

$$
T_{j, m}=\frac{2^{-(j+m) / 2}}{(j+m) !} \sum_{k=0}^{j} \sum_{l=0}^{m}\left(\begin{array}{l}
j \\
k
\end{array}\right)\left(\begin{array}{c}
m \\
l
\end{array}\right) x^{j-k} y^{m-l}\left(i \lambda \frac{\partial}{\partial x}\right)^{j}\left(-i \lambda \frac{\partial}{\partial y}\right)^{l},
$$

which is identical to the expression which is obtained for $K_{f}$ by choosing $f(x, y)=2^{-(j+m) / 2} x^{m} y^{j}$ in the construction of $(16)$, i.e. $K_{x^{m} y^{j}} \equiv T_{j, m}$.

In a similar manner the substitution

$$
f(x, y)=\lambda \exp i(m x+j y), \quad g(x, y)=\lambda \exp i(n x+k y)
$$

yields the equation

$$
\left[V_{j, m}, V_{k, n}\right]=\frac{i}{\lambda} \sin \lambda(m k-n j) V_{j+k, m+n}
$$

where now $V_{j, m}$ denotes $K_{\exp i(m x+j y)}$. This equation is isomorphic to (10). Thus there are two equivalent ways to establish the Moyal bracket algebra; either take the algebra of Weyl ordered polynomials in $P, Q$, and extend this to the algebra of functions $f(P, Q)$ defined through a formal Weyl-ordered power series; or by the association of differential operators with functions $f(x, y)$ through the ordering prescription of Eq. (15). As $\lambda \rightarrow 0$ the algebra becomes the Poisson algebra

$$
\left[L_{f}, L_{g}\right]=i L_{\{f, g\}}, \text { where } L_{f}=f_{x} \partial_{y}-f_{y} \partial x .
$$

This algebra describes area preserving diffeomorphisms of two dimensional surfaces; for the choice of exponentials for $f$ [4] it is the torus, for powers the plane [7], etc. It is important in investigations of Poisson Lie algebras, and bi-algebras with Hamiltonian structure $[3,9]$. The algebra (20) admits a central extension linear in the indices of the form $\left(c_{1} j+c_{2} m\right) \delta_{(j+k, 0)} \delta_{(m+n, 0)}$ which is compatible with the Jacobi identities.

We now repeat these investigations for the case where $P, Q$ satisfy a quantum deformation of the canonical commutations relations.

\section{Representations of $q$-Heisenberg Algebra}

Consider the algebra

$$
P Q-q Q P=i \lambda I
$$

This algebra is a canonical form for the general quadratic relation between the operators $P, Q$ and $I$, the identity element, as squares and linear terms in $P, Q$ may be transformed away by forming new linear combinations. In general the representations of (22) are infinite dimensional; however if $q^{N}=1$ for some integral 
$N$, they are of finite dimension. Let us consider the generic case first. Suppose we define a $q$-derivation through the equation

$$
\frac{d_{q}}{d x} f(x)=\frac{f(q x)-f(x)}{x(q-1)} .
$$

This satisfies the Leibniz rule

$$
\frac{d_{q}}{d x} f(x) g(x)=f(q x) \frac{d_{q}}{d x} g(x)+\left(\frac{d_{q}}{d x} f(x)\right) g(x) .
$$

(In fact, (23) may be deduced from (24) and $\frac{d_{q}}{d x} x=1$ as well as the converse.) Then (24) gives a representation of (22) in the form

$$
P=i \lambda \frac{d_{q}}{d x} ; \quad Q=x
$$

in analogy with the usual representation of the canonical commutation relations.

We now turn to the case where $q$ is an $N^{\text {th }}$ root of unity. Subdivide into two cases according as $\lambda=0$ and $\lambda \neq 0$. The case where $\lambda=0$ has already been considered in Eq. (11). Here we find two inequivalent finite dimensional representations when $\lambda \neq 0$.

It is easy to see that when $q^{N}=1$, then $P^{N}=\alpha I, Q^{N}=\beta I(\alpha, \beta$ constants $)$ are ideals of (22) since;

$$
\left[P, Q^{N}\right]=Q^{N-1}\left(1+q+q^{2}+\cdots q^{N-1}\right) \equiv 0,
$$

and similarly for $P^{N}$, so both must be proportional to the identity, or zero. Let us consider two cases

Case (1). Let $P^{N}=Q^{N}=I$. The representations may be constructed with the aid of the auxiliary matrices $g_{q}, h_{q}$;

$$
g_{q}=\left(\begin{array}{ccccc}
1 & \cdot & \cdot & \cdots & \cdot \\
\cdot & q & \cdot & \cdots & \cdot \\
\cdot & \cdot & q^{2} & \cdots & \cdot \\
\vdots & \vdots & \vdots & \ddots & \vdots \\
\cdot & \cdot & \cdot & \cdots & q^{N-1}
\end{array}\right) \quad h_{q}=\left(\begin{array}{ccccc}
\cdot & a_{1} & \cdot & \cdots & \cdot \\
\cdot & \cdot & a_{2} & \cdots & \cdot \\
\vdots & \vdots & \vdots & \ddots & \vdots \\
\cdot & \cdot & \cdot & \cdots & a_{N-1} \\
a_{N} & \cdot & \cdot & \cdots & \cdot
\end{array}\right)
$$

with

$$
\prod_{i=1}^{N} a_{i}=1
$$

The matrix $g_{q}$ is similar to $g$ in Sect. (1), except for the replacement of $\omega$ with $q$. These matrices satisfy the algebra;

Then $P, Q$, are given by

$$
h_{q} g_{q}=q g_{h} h_{q}
$$

$$
P=\frac{g_{q}^{-1}+h_{q}}{1-q}, \quad Q=g_{q} .
$$

The most general representation with $Q$ diagonal is of this form. $g_{q}$ is unitary; $h_{q}$ is unitary also if $a_{i} a_{i}^{*}=1$. It is straightforward to show using (28) that the 
commutation relations (12) are satisfied. The proof that $P^{N}$ is proportional to the identity depends upon the following

\section{Lemma}

$$
\text { Trace }\left\{P^{r}\right\}=0 \quad\{r=0,1, \ldots, N-1\} .
$$

Proof. Every term in the expansion of $P^{r}$ is either of-diagonal, or is $g^{(-r)}$. Hence it is traceless. The Cayley-Hamilton theorem for the representation of $P^{N}$ then implies $P^{N}=\alpha I$ for some $\alpha$.

A representation dependent proof of this result is straightforward, and hinges upon the subsidiary condition $\prod_{i=1}^{N} a_{i_{1}}=1$. If the right-hand side is chosen as zero then at least one $a_{i}$ vanishes, and we have a representation with $Q^{N}=1 P^{N}=0$. In fact the representation (25) is of this nature, since $\left(i \lambda \frac{d_{q}}{d x}\right)^{N}=0$ on the space spanned by the monomials $x, x^{2}, x^{3}, \ldots$ If $x$ is cyclotomic of order $N$, i.e. $x^{N}=1$, then these operators are in the class $Q^{N}=1 P^{N}=0$.

Case 2. Representations in the nilpotent case $Q^{N}=P^{N}=0$ may be expressed in the suggestive form

$$
\begin{aligned}
& P=\left(\begin{array}{ccccc}
\cdot & \sqrt{[1]} & \cdot & \cdots & \cdot \\
\cdot & \cdot & \sqrt{[2]} & \cdots & \cdot \\
\vdots & \vdots & \vdots & \ddots & \vdots \\
\cdot & \cdot & \cdot & \cdots & \sqrt{[N-1]}
\end{array}\right) \\
& Q=\left(\begin{array}{ccccc}
\cdot & \cdot & \cdots & \cdot & \cdot \\
\sqrt{[1]} & \cdot & \cdots & \cdot & \cdot \\
\cdot & \sqrt{[2]} & \cdots & \cdot & \cdot \\
\vdots & \vdots & \ddots & \vdots & \vdots \\
\cdot & \cdot & \cdot & \sqrt{ }[N-1] & \cdot
\end{array}\right) .
\end{aligned}
$$

Here $[r]$ denotes

$$
[r]=\frac{1-q^{r}}{1-q} .
$$

We see that this representation is a $q$-analogue of the usual one for creation and annihilation operators. Note that $Q$ is the transpose of $P$, rather than the Hermitian conjugate. Note also

$$
g_{q}=[P, Q] \text {. }
$$

This representation has occurred frequently in the recent literature [10].

\section{Weyl Symmetrization for $q$-Heisenberg Algebra}

There are to natural procedures for the association of an algebra of homogeneous polynomials in $P, Q$ subject to the $q$-Heisenberg commutation rule. The first is a 
direct extension of Weyl symmetrisation. Suppose first $q$ is not a root of unity. The operation which replaces this procedure for the algebra (10) is simply: Replace $T_{j, m}$ by the weighted average of all monomials of degree $j$ in $P, m$ in $Q$, the term

$$
P^{j_{1}} Q^{m_{1}} P^{j_{2}} Q^{m_{2}} \ldots P^{j_{k}} Q^{m_{l}}, \quad \sum_{r=1}^{k} j_{r}=j, \quad \sum_{r=1}^{l} m_{r}=m
$$

being weighted with a factor $q^{-\alpha}$, where

$$
\alpha=\sum_{r=1} j_{r}\left(\sum_{s=r}^{l} m_{s}\right) .
$$

In the limit as $q \rightarrow \pm 1$, this prescription ensures complete symmetrisation or anti-symmetrisation respectively. Remarkably, the same generating function which was employed in Sect. 2 admits a re-interpretation as a generating function for the ${ }_{q} T_{m, j}$ the quantum deformation of $T_{m, j}$. Recall Eq. (4);

$$
(a P+b Q)^{s}=\sum: s_{j=0}\left(\begin{array}{l}
s \\
j
\end{array}\right) a^{j} b^{s}-j T_{j, s-j} .
$$

Let us suppose now that $a, b$ are co-ordinates in a quantum plane as described by Manin [11];

$$
a b=q^{-1} b a,
$$

and let us maintain the commutation

$$
[a P, b Q]=i \lambda a b .
$$

We also assume that $a, b$ commute with $P, Q$. Then (34), (35) imply

$$
P Q-q Q P=i \lambda
$$

and the generating function for the quantum deformation ${ }_{q} T_{j, m}$ is

$$
(a P+b Q)^{s}=\sum_{j=0}^{s} \frac{[s] !}{[j] ![s-j] !} a^{j} b^{s}-j_{q} T_{j, s-j} .
$$

Here $[s]$ ! means $[s][s-1] \cdots[10]$ and does not vanish for $s$ positive as $q$ is not a root of unity.

The operators ${ }_{q} T_{j, m}$ satisfy the deformed commutator

$$
\begin{aligned}
& q^{(k m-j n) / 2}{ }_{q} T_{j, m q} T_{k, n}-q^{(j n-k m) / 2}{ }_{q} T_{k, n q} T_{j, m} \\
& =\sum_{r=0}^{\min \{[(j+k-1) / 2],[(m+n-1) / 2]\}}(2 i \lambda)^{2 r+1} \\
& \cdot \sum_{s=0}^{2 r+1}(-1)^{s} \frac{[j] ![k] ![m] ![n] !}{[2 r+1-s] ![s] ![j+s-2 r-1] ![k-s] ![m-s] ![n+s-2 r-1] !} \\
& { }_{q} T_{j+k-2 r-1, m+n-2 r-1} \text {. }
\end{aligned}
$$

This is the quantum deformation of (5).

The crucial observation is that the operators $a P$ and $b Q$ satisfy (32). The only difference in the derivation of (37) from that of (5) therefore lies in the fact that 
the parameters of $P$ and $Q$ satisfy (31) instead of commuting. This means that in pulling out factors, of $a, b$ from (36) for example, $q$-binomial rather than binomial expressions occur. This is the reason also for the appearance of the exponential factors on the left of (37), and accounts for the replacement of the normalisation factors $j !, k$ ! etc on the right of (5). These arguments have to be considered more carefully in the case where $q$ is an $N^{\text {th }}$ root of unity $N \neq 1$, as $[n]$ ! vanishes for $n \geqq N$. In this case there are finitely many distinct operators ${ }_{q} T_{j, m}, N^{2}$ of them, in fact, if the finite representations of (22) are chosen. They close under $q$-commutation as in (37), with the modification that any term on the right with index $j+k-2 r-1$, or $m+n-2 r-1 \geqq N$ can appear. This algebra is a $q$-deformation of $U(N)$, with $q^{N}=1$. On the other hand a commutator algebra can also be constructed from the same operators $P, Q$ which is isomorphic to $U(N)$, since this algebra may also be realised in terms of operators constructed as in Sect. 2 from $g_{q}, h_{q}$ by

$$
J_{j, m}=q^{j m / 2} g_{q}^{j} h_{q}^{m}, \quad 0 \leqq j, m \leqq N .
$$

This realisation does not lead to a description of the generators in terms of homogeneous polynomials in $P, Q$ however. The relation between those two algebras deserves further consideration.

\section{The Derived Series}

The second way to construct a polynomial algebra which also gives rise to homogeneous polynomials is to consider the derived series of repeated Lie brackets of $P, Q$, which is closed under the bracket operation. This set of polynomials, modulo the equivalence relations;

$$
\begin{aligned}
& \sqrt{\frac{1}{q}} P^{2} Q-\left(\sqrt{q}+\sqrt{\frac{1}{q}}\right) P Q P+\sqrt{q} Q P^{2} \equiv 0, \\
& \sqrt{\frac{1}{q}} P Q^{2}-\left(\sqrt{q}+\sqrt{\frac{1}{q}}\right) Q P Q+\sqrt{q} Q^{2} P \equiv 0
\end{aligned}
$$

generates an algebra which vanishes in the limit $q \rightarrow 1$.

Again there is a distinction between the case where $q$ is a root of unity other than once, and the generic case. In the former case the fact that there are finite dimensional representations gives a finite algebra isomorphic to $S U(N)$ when $P, Q$ are nilpotent, and $U(N)$ otherwise. It possesses a subalgebra consisting of $N(N+1) / 2$ operators, represented here by upper triangular matrices, generated by the closure of $P Q-Q P, P$ with $P^{N}=1$ under commutation.

\section{The $q$-Exponential}

There exists the interesting possibility of a double quantisation, by imposing $\lambda=\frac{\pi}{M}$ upon the commutation relations (22), in addition to the possibility of choosing $q$ 
a root of unity. As a step towards the construction of a quantum version of the Moyal bracket consider the $q$-analogue exponential function;

$$
\begin{aligned}
& \exp _{q}(P)=\sum_{0}^{\infty} \frac{P^{n}}{[n] !}, \\
& \exp _{q}(P) \times \exp _{1 / q}(-P)=1 .
\end{aligned}
$$

Equation (41) is a special case of the following;

Theorem.

$$
\exp _{1 / q}(-a P) \exp _{q}(b Q) \exp _{q}(a P)=\exp _{q}(-i a b \lambda) \exp _{q}(b Q)
$$

Proof. Since

$$
\left[\frac{(a P)^{n}}{[n] !}, b Q\right]=\frac{(a P)^{n-1}}{[n-1] !} i a b \lambda .
$$

(Here $P, Q$ and $a, b$ obey the commutation relations $(22,34)$.)

$$
\left[\exp _{q}(a P), b Q\right]=\exp _{q}(a P) a b i \lambda
$$

i.e.

$$
\exp _{1 / q}(-a P) b Q \exp _{q}(a P)=i a b \lambda+b Q
$$

Denoting the left-hand and right-hand sides of this equation by $L, R$ respectively, from $\sum \frac{L^{n}}{[n] !}=\sum \frac{R^{n}}{[n] !}$. This gives (42).

Since $b$ may be scaled, the limit $b \rightarrow 0$ may be taken in (42). This yields (41). Rearranging (42) we obtain

$$
\exp _{q}(b Q) \exp _{q}(a P)=\exp _{q}(a P) \exp _{q}(-i a b \lambda) \exp _{q}(b Q) .
$$

This is nothing but a relation of the form (10). Note also the commutation,

$$
\exp _{q}(-i a b \lambda) \exp _{q}(b Q)=\exp _{q}(-i a b \lambda+b Q) .
$$

The quantum analogue of the Baker-Campbell-Hausdorff identity (7), is obtained as follows: introduce a second quantum plane parametrised by $\alpha, \beta$ with

$$
\alpha \beta=\frac{1}{q^{2}} \beta \alpha .
$$

The $\alpha, \beta$ may be represented by $a, b^{2}$ respectively. Then the identity is

$$
\begin{aligned}
\exp _{1 / q}(\alpha P+\beta Q) & =\exp _{1 / q}(\beta Q) \times \exp _{1 /\left(q^{2}\right)}\left(\frac{\beta \alpha i \lambda}{q(1+q)}\right) \times \exp _{1 / q}(\alpha P), \\
\exp _{q}(\alpha P+\beta Q) & =\exp _{q}(\alpha P) \times \exp _{q^{2}}\left(\frac{\alpha \beta i \lambda}{q(1+q)}\right) \times \exp _{q}(\beta Q) .
\end{aligned}
$$

Proof. Consider the differential operator

$$
\frac{d_{q^{-1}}}{d x} \exp _{1 / q}(\beta Q x) \times \exp _{1 /\left(q^{2}\right)}\left(\frac{\beta \alpha \lambda x^{2}}{q(1+q)}\right) \times \exp _{1 / q}(\alpha P x)
$$




$$
\begin{aligned}
= & \beta Q \exp _{1 / q}(\beta Q x) \times \exp _{1 /\left(q^{2}\right)}\left(\frac{\beta \alpha \lambda x^{2}}{q(1+q)}\right) \times \exp _{1 / q}(\alpha P x) \\
& +\exp _{1 / q}\left(\beta \frac{Q x}{q}\right) \times \frac{\beta \alpha \lambda x}{q} \exp _{1 /\left(q^{2}\right)}\left(\frac{\beta \alpha \lambda x^{2}}{q(1+q)}\right) \times \exp _{1 / q}(\alpha P x) \\
& +\exp _{1 / q}\left(\beta \frac{Q x}{q}\right) \times \exp _{1 /\left(q^{2}\right)}\left(\beta \alpha \lambda\left(\frac{x^{2}}{q(1+q)}\right) \times \alpha P \exp _{1 / q}(\alpha P x) .\right.
\end{aligned}
$$
By use of the commutation relations the factors $\frac{\beta \alpha \lambda x}{q}$ and $\alpha P$ may be moved to
the left. The terms linear in $x$ cancel leaving

$$
\begin{aligned}
& \frac{d_{q^{-1}}}{d x} \exp _{1 / q}(\beta Q x) \times \exp _{1 /\left(q^{2}\right)}\left(\frac{\beta \alpha \lambda x^{2}}{q(1+q)}\right) \times \exp _{1 / q}(\alpha P x) \\
& \quad=(\alpha P+\beta Q) \exp _{1 / q}(\beta Q x) \times \exp _{1 /\left(q^{2}\right)}\left(\frac{\beta \alpha \lambda x^{2}}{q(1+q)}\right) \times \exp _{1 / q}(\alpha P x)
\end{aligned}
$$

But this is just the $q$-difference equation for a $q^{-1}$-exponential, hence the relation (49) follows, the arbitrary factor of integration being fixed by choice of $x=1$. The second relation is just the inverse of this.

\section{Remarks}

There are many hints that the extention of $q$-analysis to many variables requires the notion of non-commuting co-ordinates, or at least is facilitated by their introduction. In a generalisation of (17), for example, it is natural to replace the derivatives in the representation of $P, Q$ by their quantum analogue difference operators and this necessitates that the corresponding independent variables $x, y$ be treated as non-commuting operators in order that the commutation relations (22) should be fulfilled. Consider the quantum analogue difference operators denoted by

$$
\frac{\partial_{q^{-1}}}{\partial x} ; \quad \frac{\partial_{q}}{\partial y}
$$

and defined by

$$
\begin{aligned}
\frac{\partial_{q^{-1}}}{\partial x} f(x, y) & =\left(\frac{1}{x}\right) \frac{q\left(f\left(\frac{x}{q}, y\right)\right)-f(x, y)}{1-q} \\
\frac{\partial_{q}}{\partial y} f(x, y) & =\left(\frac{1}{y}\right) \frac{f(x, q y)-f(x, y)}{q-1}, \text { where } x y=q y x .
\end{aligned}
$$

These satisfy the Leibniz rules;

$$
\frac{\partial_{q^{-1}}}{\partial_{x}} f(x, y) g(x, y)=\left(\frac{\partial_{q^{-1}}}{\partial x} f(x, y)\right) g\left(\frac{x}{q}, y\right)+f\left(x, \frac{1}{q} y\right)\left(\frac{\partial_{q^{-1}}}{\partial_{x}} g(x, y)\right)
$$




$$
\begin{aligned}
\frac{\partial_{q}}{\partial y} f(x, y) g(x, y) & =\left(\frac{\partial_{q}}{\partial y} f(x, y)\right) g(x, q y)+f(q x, y)\left(\frac{\partial_{q}}{\partial y} g(x, y)\right) \\
\frac{\partial_{q-1}}{\partial x} x^{n} & =[n]\left(\frac{x}{q}\right)^{n-1} \\
\frac{\partial_{q}}{\partial y} y^{n} & =[n] y^{n-1} .
\end{aligned}
$$

Note the differentiation with respect to $x$ is with the parameter $\frac{1}{q}$, whereas that with respect to $y$ is with parameter $q$. This is necessary in order that the following equations are valid. Then, these Leibniz relations impose

$$
\frac{\partial_{q^{-1}}}{\partial x} \frac{\partial_{q}}{\partial y}=\frac{1}{q} \frac{\partial_{q}}{\partial y} \frac{\partial_{q^{-1}}}{\partial x}
$$

and at the same time, preserve the commutation relations (22) in the identification

$$
P=\frac{1}{\sqrt{1+q}}\left(x-i \lambda \frac{\partial_{q}}{\partial y}\right), \quad Q=\frac{1}{\sqrt{1+q}}\left(y+i \lambda \frac{\partial_{q^{-1}}}{\partial x}\right) .
$$

Thus the considerations of classical function theory must be extended for functions of more than one variable to functions on associative, but not necessarily commutative algebras when $q$-analogoues of classical results are sought. There remain many unanswered questions concerning the second deformation of the Moyal and Poisson brackets. We hope to return to those questions at a later time.

Recent work on finite dimensional Lie algebras has included the $N \rightarrow \infty$ limit of the Zamolodchikov $W_{N}$ algebras [11]. This admits an interpretation also in terms of area preserving deformations $[2,12]$ and a deformation which may be cast in Moyal form $[13,14]$.

Acknowledgements. We should like to thank P. Fletcher, D. Kazhdan and C. K. Zachos for discussions, and D. Fairlie wishes to acknowledge the hospitality of Argonne National Laboratory.

\section{References}

1. Weyl, H.: The theory of groups and quantum mechanics (1932) (reprinted Dover Publications)

2. Moyal, J.: Proc. Camb. Phil. Soc. 45, 99-124 (1949); Baker, G.: Phys. Rev. 109, 2198-2206 (1958); Fairlie, D. B.: Proc. Camb. Phil. Soc. 60, 581-586 (1864)

3. Gelfand, I. M., Dorfman, I. Ya.: J. Funct. Anal. Appl. 13, 248 (1980); 14, 248 (1980); 15, 173 (1981); 16, 241 (1982). (Russian originals; Funksional'ni Analizi i ego Prilozhenia 13, (4) 13-3? (1980); 14, (3) 17-74 (1980) 15, (3) 23-40 (1981); 16, (4) 1-9 (1982)

4. Fairlie, D. B., Fletcher, P., Zachos, C. K.: Phys. Lett. B218, 203 (1989); J. Math. Phys. 31, 1088 (1990); Fairlie, D. B., Zachos, C. K.: Phys. Lett. B224, 101 (1989)

5. Hoppe, J.: MIT thesis (1982)

6. Bakas, I.: The Structure of the $W_{\infty}$ algebra, U. Maryland UMPP 90-085

7. Bender, C. M., Dunne, G.: Phys. Rev. D40, 3504 (1989)

8. Bayen, F., Flato, M., Fronsdal, C., Lichnerowicz, A., Sternheimer, D.: Ann. Phys. N.Y. 111, 61, 111 (1978); Averson, A.: Commun. Math. Phys. 89, 77-102 (1983); Estrada, R., GarciaBondia, J. M., Varilly, J. C.: J. Math. Phys. 30, 2789 (1989) 
9. Drinfeld, V. G.: Sov. Math. Doklady 27, 68 (1983)

10. Macfarlane: A. J.: J. Phys. A22, 4581 (1989); Biedenharn, L. C.: J. Phys. A22, L873 (1989); Floratos, E. C.: Phys. Lett 233B, 395 (1989)

11. Manin, Yu. I.: Annales de L'Institute Fourier, Grenoble 37, 191 (1987); Commun. Math. Phys. 123, 163 (1989)

12. Zamolodchikov, A. B.: Teo. Mat. Fiz. 65, 347 (1985); Fateev, V. A., Lykyanov, S.: Int. J. Mod. Phys. A3, 507 (1988)

13. Pope, C. N., Romans, L. J., Shen, X.: $W_{\infty}$ and the Racah-Wigner algebra, preprint, CTP TAMU-72/89, USC-89/HEP040

14. Bakas, I., Kiritsis, E.: Bosonic realisation of a universal $W$-algebra and $Z_{\infty}$ parafermions, preprint, LBL-28714, UCB-PTH-90/8, UMD-PP90-160

Communicated by A. Jaffe 
